\title{
Métodos de valuación de externalidades ambientales provocadas por obras de ingeniería
}

\author{
Alberto Jaime P. y R.0. Tinoco-López \\ División de Ingenierías Civil y G eomática, Facultad de Ingeniería, UN AM \\ E-mail: ajaimep@ cancun.fi-a.unam.mx \\ (recibido: febrero de 2005; aceptado: septiembre de 2005)
}

\begin{abstract}
Resumen
El objetivo de este trabajo es analizar y aplicar los métodos de evaluación económica de externalidades ambientales en proyectos de ingeniería civil. Para esto se requiere, tanto el conocimiento de las técnicas de evaluación de impacto ambiental, como los modelos para evaluar las variables ambientales de modo que puedan identificarse plenamente las consecuencias que estos generan. De esta manera, se observarán los efectos positivos y negativos derivados por dichos impactos y así podrá realizarse su evaluación económica
\end{abstract}

Descriptores: Externalidades ambientales, evaluación ambiental, bienes y servicios ambientales

\begin{abstract}
Theobjectiveof this ar ti cleis to analizeand ap ply theassess mentmeth ods ofen vi ron $m$ en tal exter nali. ties in civil en gi neer ing pro jects. In or der to do that, theknowl edge of en vi ron men tal impactassessment meth ods, aswell asproceduresfor iden ti fication and val u a tion of en vi ron men tal vari ables is required $s 0$ a com pleteiden ti $\mathrm{fi}$ ca tion of im pacts and prediction of con sequences can bedone. By do ing this, both posi itiveand neg a tiveef fects of $i m$ pacts could beas sessed en vi ron men tally and economi cally.
\end{abstract}

Keywords: En vi ron men tal exter nali ties, en vi ron men tal assess ment, en vi ron men tal goodsand services.

\section{Introducción}

Los efectos que puede ocasionar una obra civil en el medio, deben ser identificados y evaluados desde la etapa de planeación. Esto tiene por objetivo cuantificar los costos y beneficios externos de una obra, que son aquellos que tarde o temprano tendrá que pagar o disfrutar la sociedad en su conjunto como consecuencia de los efectos provocados por la obra en el medio (Ottinger y Wooley, 1990).

Las externalidades se producen cuando las actividades sociales o económicas de un grupo de personas tienen un impacto sobre otro (el ambiente se considera como un grupo más), y dicho impacto no está plenamente tomado en cuenta por el primer grupo (European Commission, 2003).

Otra forma de ver las externalidades (Meade, 1973), es como eventos que confieren beneficios o costos considerables a una persona o grupo de personas sin que éstas hayan dado su consentimiento al momento de tomar las decisiones que llevaron directa 0 indirectamente a la ocurrencia de dichos eventos. Visto de otra manera (Baumol y Oates, 1988), una externalidad se presenta cuando la utilidad de un individuo A se ve afectada por ciertas variables cuyos valores son decididos por otros (personas, corporaciones o gobiernos), sin interesarse en los efectos y el bienestar del individuo $A$. 
Métodos de valuación de externalidades ambientales provocadas por obras de ingeniería

En general, las externalidades ambientales pueden ser: efectos en el paisaje, efectos en la salud humana (morbilidad), incremento o disminución de muertes (mortalidad), pérdida del equilibrio ecológico, efectos en los acuíferos y cuerpos de agua superficiales, cambios en la calidad del aire, cambios en el nivel de calidad del agua, pérdida de sitios recreativos, efectos en suelos o calentamiento global. Algunos de estos costos pueden estimarse cuantitativamente en términos monetarios (pérdida de cosechas, bosques o tierras cultivables); mientras que otros no (pérdida del paisaje nat u ral o incremento en la morbilidad), aunque de estos se puede aspirar a tener una idea cualitativa y suficientemente objetiva (Jaime, 2003).

Para esto se requiere, tanto el conocimiento de las técnicas de evaluación de impacto ambiental, como los modelos para evaluar las variables ambientales de modo que puedan identificarse plenamente las consecuencias que estos generan. De esta manera, se observarán los efectos tanto positivos como negativos debidos a dichos impactos y podrán expresarse en términos económicos.

\section{Costos de las obras}

\section{Costos directos}

Los costos de las obras se pueden dividir en costos directos e indirectos. Entre estos últimos están los costos externos ambientales. Todos los gastos necesarios para llegar al inicio de la operación de la obra son los costos directos del proyecto, que incluyen un in tan gi ble, el costo por financiamiento, es decir, los intereses que se deben pagar hasta ese momento por el dinero empleado. Este intangible es función del tiempo, cualquier retraso en los programas de obra y de puesta en marcha incrementan su monto.

Cabe aclarar que las obras de ingeniería civil, por ejemplo, tienen generalmente diversas alternativas para realizarse. Así, la localización de la trayectoria de una carretera puede incluir varias opciones en todo su trazo o por tramos, de esta forma, se puede decidir disminuir la longitud de la carretera colocando un puente o haciendo un túnel; en cuanto a las presas, comúnmente se tienen varias opciones señaladas por las boquillas geológicas más aptas para el proyecto. Las técnicas de selección de sitios para instalar parques industriales, grandes plantas de generación de energía eléctrica, plantas cementeras o de otros tipos, toman en cuenta para su análisis diversas alternativas, en función de aspectos económicos, sociales, políticos y de infraestructura, tales como: vías de acceso, poblaciones cercanas, grado de desarrollo económico y social de la zona de influencia del proyecto, disponibilidad de energéticos y agua, etc.

A partir de la década de los 90 , a los análisis de alternativas se le añaden los costos externos ambientales, debido a los efectos en el ambiente producidos por cada uno de ellos. Dichos efectos deben ser identificados y evaluados económicamente para poder hacer un análisis completo beneficio-costo de las distintas alternativas de un proyecto, y así elegir la óptima, tomando en cuenta los costos externos ambientales. Este ejercicio se denomina internalización de costos externos.

Como parte del análisis financiero, se considera que du rante la operación de la obra debe redituarse el cap i tal empleado (costos directos), el costo financiero del mismo (du rante esta etapa), los costos de operación y mantenimiento, así como los beneficios o utilidades esperadas en el período de vida útil. A esto debe añadirse algo que muy frecuentemente se deja de lado, la creación de un fondo económico para el desmantelamiento de la obra al final de su vida útil.

\section{Externalidades ambientales}

Las externalidades ambientales deben ser parte in te gral en los análisis beneficio-costo al momento de evaluar las diferentes alternativas de un proyecto de obra civil. Du rante la planeación y en la etapa de selección del sitio o trayectoria de la obra (Jaime, 2003), para cada alternativa, hay que estimar los costos directos de la obra, más los costos de las externalidades, para optar por aquella cuya relación beneficio-costo sea la adecuada. Suele ocurrir que en un sitio o trayectoria, los costos directos son menores que en otros; sin embargo, las externalidades son más elevadas que en estos últimos. Al sumar los costos directos más las externalidades, se tiene la visión 
completa del problema y se puede elegir el sitio 0 trayectoria más apropiado en términos ambientales, sociales y económicos, a mediano y largo plazos.

Uno de los principales problemas al hablar de costos externos, se inicia al buscar un punto óptimo entre la magnitud del impacto que se produce y el costo que representaría a una empresa evitarlo o mitigarlo. La complejidad del tema re side en la repartición del costo, dado que puede existir un óptimo para la empresa que no necesariamente satisfaga al resto de la sociedad y viceversa.

En este aspecto, existen leyes, reglamentos y normas, que nos marcan el proceso para realizar los estudios de impacto ambiental y la preparación de las Ilamadas Manifestaciones de Impacto Ambiental. En el estudio de externalidades ambientales, dicha normatividad se toma en cuenta; sin embargo, este trabajo se enfoca al análisis y aplicación de los métodos de valoración económica de externalidades para su posterior internalización, esto es, incluirlas como costo directo en el proyecto definitivo, y como elemento de decisión en el análisis beneficio-costo de las diferentes alternativas.

La evaluación de externalidades nos permite entre otras cosas:

- Comparar la magnitud de diferentes impactos ambientales en términos económicos, que es el lenguaje más empleado por el tomador de decisiones.

- Comparar los impactos ambientales negativos totales contra los positivos.

- Proveer las bases para un criterio claro y objetivo que permita al decisor aceptar 0 rechazar un proyecto. Además, poder presentar a los grupos de interés la valía de la obra en términos ambientales.

- Considerar la variable ambiental junto con las técnicas financieras, sociales y políticas dentro del análisis de un proyecto desde su etapa de planeación.

Las externalidades ambientales son de carácter relativo, tanto en el espacio como en el tiempo.
Algunos factores y efectos pueden ser considerados adversos en ciertas regiones y benéficos en otras, además, la percepción que se tiene de ellos puede cambiar con el tiempo, por lo que este tipo de costos y beneficios externos no pueden estudiarse de manera aislada, sino en un marco de contexto so cial, geográfico, histórico, cul tural y del tiempo, en el cual se proponen llevar a cabo.

\section{Identificación y evaluación de impactos ambientales}

La evaluación ambiental debe ser parte del proceso para decidir sobre el proyecto o actividad que un promotor desea llevar a cabo. Es por tanto, imprescindible que el promotor tome en cuenta desde el principio a la variable ambiental, así como lo hace con los aspectos técnicos, financieros, de oportunidad, etc., (Jaime, 2003).

Si la evaluación ambiental se hace sobre un proyecto ya decidido, la vari able ambiental se agrega como un pegote que lo desfigura, y no deja satisfechos ni al tomador de decisiones ni a los usuarios, así como tampoco a las comunidades afectadas. Pero sobre todo, se pierde la oportunidad de dar soluciones integrales a ciertos impactos ambientales con un bajo costo, es decir, dichas soluciones se pueden incluir en la concepción misma del proyecto con ahorros en dinero, tiempo y forma. Además de prevenir algunos impactos que no será necesario remediar.

El procedimiento para la evaluación de impactos ambientales puede establecerse en varias etapas de acuerdo con el reglamento de la LGEEPA en materia de impacto ambiental. Una primer etapa consiste en:

1) Detallar las características del proyecto, de las obras y actividades involucradas en cada una de sus fases.

2) Caracterizar la situación ambiental existente y los posibles niveles de alteración.

3) Describir en general, los aspectos ambiental y socioeconómico; y 
Métodos de valuación de externalidades ambientales provocadas por obras de ingeniería

4) Predecir las condiciones ambientales que se tendrían de no llevarse a cabo el proyecto.

En una segunda etapa, se incluye la identificación, predicción y evaluación de los efectos que tendrá la implantación del proyecto. Para esto, se cuenta con diversas técnicas y modelos de análisis.

La tercera etapa consiste en proponer medidas de prevención y mitigación de los efectos negativos que ocasionaría el proyecto sobre el ambiente, considerando los impactos identificados en la segunda etapa. De esta manera, se elabora una Manifestación de impacto ambiental, que contiene los siguientes puntos:

- Datos generales del proyecto, del promovente y del responsable del estudio de impacto ambiental.

-. Descripción del proyecto.

- Vinculación con los ordenamientos jurídicos aplicables en materia ambiental y, en su caso, con la regulación sobre uso del suelo.

- Descripción del sistema ambiental y señalamiento de la problemática ambiental detectada en el área de influencia del proyecto.

- Identificación, descripción y evaluación de los impactos ambientales.

- Medidas preventivas y de mitigación de los impactos ambientales.

- Pronósticos ambientales y, en su caso, evaluación de alternativas.

- Identificación de los instrumentos metodológicos y elementos técnicos que sustentan la información señalada en las fracciones anteriores.

Para identificar, predecir y evaluar los efectos ambientales de las obras, se cuenta con diversas técnicas y modelos de análisis. Se puede recurrir a listas de verificación, diagramas de flujo y redes, panel de expertos, carto grafía ambiental, ma tri ces causa-efecto, etc.

Las listas de chequeo consisten en listas exhaustivas para identificar los impactos. Existen varios tipos, desde puramente indicativas hasta cuantitativas, las cuales emplean procedimientos estándar para detectar los principales impactos. Éstas tienen por objeto, identificar todas las consecuencias posibles ligadas a las acciones que se tiene previsto ejecutar, por lo que es fun da men tal no omitir alguna alteración relevante durante su elaboración. Se cuenta con listados simples, descriptivos, escalonados e incluso cuestionarios. Un ejemplo puede verse en la figura 1.

Los diagramas de flujo permiten identificar impactos indirectos, establecen relaciones de causalidad, generalmente lineal, entre la acción propuesta y el elemento afectado. También son usados para la discusión de impactos directos y su aplicación se vuelve compleja al multiplicarse las acciones e impactos involucrados, como puede verse en la figura 2, es por eso que se opta por esta técnica sólo cuando existe cierta simplicidad en los impactos. Los listados y los diagramas de flujo con frecuencia se emplean juntos.

Por otro lado, la técnica de panel de expertos consiste en la sistematización de las consultas a grupos de expertos familiarizados con un proyecto 0 con temas especializados y está en función del tipo de especialistas disponibles. Más que definir parámet ros específicos para aspectos a considerar en un futuro, esta técnica identifica una amplia gama de impactos, establece medidas de mitigación y permite establecer métodos de seguimiento y con trol.

La cartografía ambiental involucra elementos como fotografías aéreas, mapas topográficos, observaciones en terreno y opinión de expertos, entre otros. Uno de estos procedimientos consiste en la superposición de transparencias, figura 3, que ubican impactos individuales sobre el mapa de un territorio, con el fin de obtener un impacto total. Permite analizar proyectos puntuales como edificios, presas o puentes; lineales, tales como carreteras, ferrocarriles, ductos, ca na les, líneas de transmisión; y superficiales: complejos industriales, embalses, centros urbanos y turísticos, entre otros. 


\section{Alberto Jaime P. y R.O. Tinoco-López}

\begin{tabular}{|c|c|}
\hline Agente & Posible impacto \\
\hline 1. Personal & $\begin{array}{l}\text { Caza, pesca, alleración de la vida de la } \\
\text { comunidad, alectación ecuncrrica, } \\
\text { desechos generados, campamentos. }\end{array}$ \\
\hline 2. Maquinaria & $\begin{array}{l}\text { Ruidos, emisiones contsminantes, aceites } \\
\text { y grasas de desecho, talleres de } \\
\text { mantenimiento y roparacion. }\end{array}$ \\
\hline $\begin{array}{l}\text { 3. Métodos de construoción } \\
\text {-uso de explosivos } \\
\text {-muros milan } \\
\text {-hincexdo de piloles } \\
\text {-martillos neurnáticos de } \\
\text { luneleo }\end{array}$ & $\begin{array}{l}\text { Polvo del frente de ataque, ruico } \\
\text { vibraciones, manejo de explosivos, } \\
\text { disposición del material de rezaga, } \\
\text { disposición de locos, alectexión de la } \\
\text { calidad del agua de cuerpos receptores, } \\
\text { alteración de manantiales y cuerpos de } \\
\text { agua, emisiones contaminantes a la } \\
\text { atmosfera, modificaciones topográficas y } \\
\text { topobalimétricas que puoden alterar el } \\
\text { habitat y la vida de las comunidades. }\end{array}$ \\
\hline 4. Bomben & $\begin{array}{l}\text { Cambias de régimen geohidrald́gica que } \\
\text { puede alectar manantiales y pozos de la } \\
\text { región. }\end{array}$ \\
\hline 5. Imyocciones & $\begin{array}{l}\text { Cambio de régimen gouhidrolćxjico, posible } \\
\text { afectación de la calidad del agua } \\
\text { subterránea. }\end{array}$ \\
\hline 6. Bancos de prestamo & $\begin{array}{l}\text { Alteración del paisaje, afectación a flora y } \\
\text { Fanna, perdida de hábitaL. }\end{array}$ \\
\hline 7. Tiraderos & $\begin{array}{l}\text { Alteración del paisaje, afectación de flora y } \\
\text { fauna, pardida de habitat. alteración del } \\
\text { drenaje natural. }\end{array}$ \\
\hline 8. Camincs de acceso & $\begin{array}{l}\text { Alteración del paisaje, afectación a flord y } \\
\text { fauna. }\end{array}$ \\
\hline 9. Portales de tùneles & Alteración del paisaje y del hábitat. \\
\hline
\end{tabular}

Figura 1. Ejemplo de listado simple

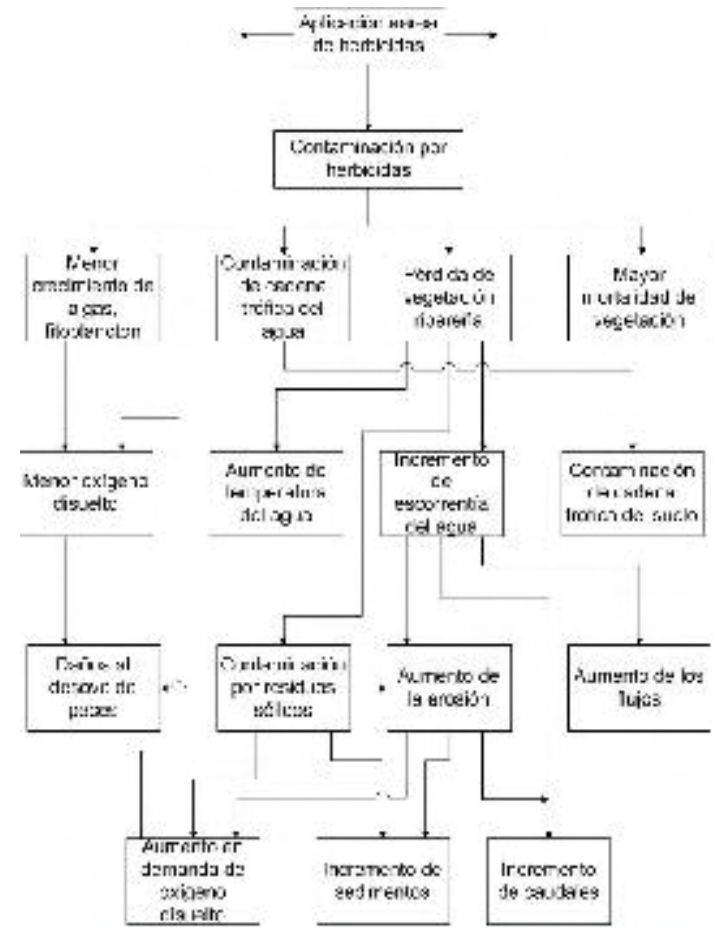

Figura 2. Ejemplo de una red 
Métodos de valuación de externalidades ambientales provocadas por obras de ingeniería

Las ma tri ces causa-efecto relacionan los impactos ambientales con las acciones de los proyectos. Consisten en un listado de acciones humanas y uno de indicadores de impacto ambiental, relacionados en forma matricial (Figura 4). Esta técnica es muy útil para identificar el origen de ciertos impactos, pero presenta problemas para definir impactos secundarios, establecer interacciones y realizar consideraciones temporales o espaciales. Estas técnicas permiten identificar, evaluar y monito rear los impactos.

Se requiere además, de modelos para cuantificar los cambios en las vari ables ambientales de interés, información in dis pens able para valorar las consecuencias de dichos cambios. Así por ejemplo, se cuenta con modelos para evaluar las modificaciones provocadas por una obra en la calidad del agua, calidad del aire, de- gradación de suelos, así como flora y fauna.

\section{Evaluación de externalidades}

La evaluación de externalidades se puede llevar a cabo con métodos directos, indirectos y de otro tipo (Tabla 1). Los métodos directos, de preferencias expresadas, consisten básicamente en preguntar directamente a los posibles interesados sobre el valor que para ellos tiene un bien o servicio ambiental. Los métodos indirectos, de preferencias reveladas, estiman el valor ambiental a partir del comportamiento observado de la población involucrada.

Tabla 1. M étodos de evaluación

\section{Métodos Directos}

\begin{tabular}{|c|c|}
\hline De valoración contingente & $\begin{array}{l}\text { Estima el valor de prácticamente cualquier servicio ambiental. Pregunta cuánto se está dispuesto apagar por } \\
\text { los servicios ambientales concretos. }\end{array}$ \\
\hline De selección contingente & $\begin{array}{l}\text { Infiere valores mediante las elecciones que realiza el entrevistado al presentársele distintas opciones de } \\
\text { intercambio }\end{array}$ \\
\hline \multicolumn{2}{|r|}{ MétodosIndirectos } \\
\hline Del costo de viaje & $\begin{array}{l}\text { Estima el valor de beneficios ambientales de un sitio, asumiendo que dicho valor se refleja en cuánto pagan } \\
\text { los individuos por acceder al sitio. }\end{array}$ \\
\hline Del precio hedónico & $\begin{array}{l}\text { Supone que la utilidad de un bien o servicio, está en función de sus atributos. Trata de separar los efectos } \\
\text { de dichos atributos para mostrar cómo sus cambios afectan en la utilidad. }\end{array}$ \\
\hline Del precio de mercado & $\begin{array}{l}\text { Estima los valores económicos de bienes o servicios ambientales que son comprados y vendidos en } \\
\text { mercados comerciales. }\end{array}$ \\
\hline \multicolumn{2}{|r|}{ Otros métodos } \\
\hline De dosis-respuesta & $\begin{array}{l}\text { Estima el impacto físico qye genera un cambio en el ambiente sobre un receptor. Establece un vínculo entre } \\
\text { un nivel de alteracion en el ambiente y un impacto físico }\end{array}$ \\
\hline $\begin{array}{l}\text { De costo de reemplazo, } \\
\text { costo de daño evitado y } \\
\text { costo del sustituto }\end{array}$ & $\begin{array}{l}\text { Estima valores económicos con pase en el costo que implica evitar los daños resultantes de la pérdida de } \\
\text { servicios ambientales, el costo de reemplazar dichos servicios o proveer servicios sustitutos. }\end{array}$ \\
\hline
\end{tabular}

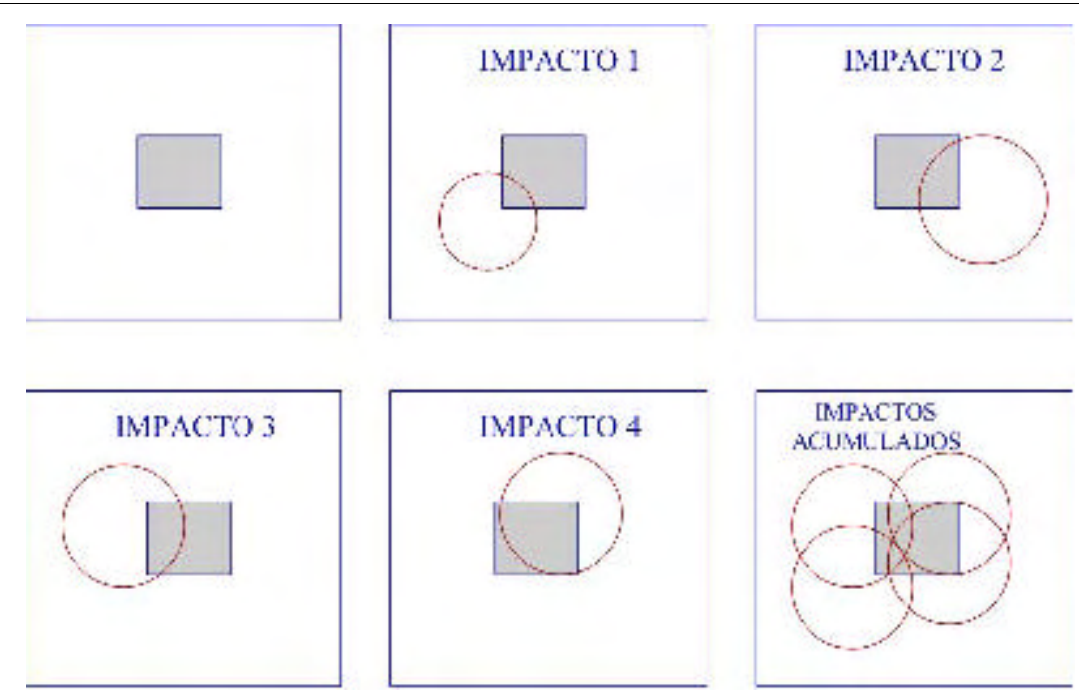

Figura 3. Cartografía ambiental 
Alberto Jaime P. y R.O. Tinoco-López

\begin{tabular}{|c|c|c|c|c|c|}
\hline $\begin{array}{l}\text { Impacto } \\
\text { ambiental }\end{array}$ & & Diseño & Construcción & Operación & Abandono \\
\hline \multirow[t]{2}{*}{ Aire } & Calidad & A & A & 1 & A \\
\hline & Ruvido & A & A & A & A \\
\hline \multirow[t]{2}{*}{ Agua } & Calidad & A & A & A & 1 \\
\hline & Canlidad & A & 1 & A. & A \\
\hline \multirow[t]{2}{*}{ Stuelo } & Frosión & A & 1 & c & A \\
\hline & Pruductividad & A & I & c & A \\
\hline \multirow[t]{2}{*}{ Flora } & Abunclancia & A & 1 & C & A \\
\hline & Representatividad & $\Lambda$ & 1 & c & c \\
\hline \multirow[t]{2}{*}{ Fauna } & Abundancia & A & 1 & 1 & A \\
\hline & Representatividad & A & 1 & 1 & $A$ \\
\hline \multirow[t]{2}{*}{ Paisaje } & Belleza & A & i & A & 1 \\
\hline & Visual & A & i & A & A \\
\hline \multirow[t]{2}{*}{ Población } & Relocalización & A & c & c & c \\
\hline & Costumbres & A & C & C & c \\
\hline Otros & Eccsistemas & A & A & A & C \\
\hline
\end{tabular}

Calificación de impacto: Inaceptable: I Crilico: C Aceptable: A

Figura 4. Ejemplo de una matriz causa-efecto

\section{Métodos directos (de preferencias expresadas)}

M étodo de valoración contingente

Este método sirve para estimar el valor de prácticamente cualquier servicio ambiental. Se en- tiende como servicio ambiental aquel beneficio o satisfactor que brinda el ambiente. Puede ser proporcionar un producto (plantas medicinales, captura de CO, madera, etc.), hasta el sentimiento de satisfacción por poder disfrutar de lo que ofrece (paisajes, solaz para el espíritu, etc.). En la figura 5 se muestra un diagrama de flujo del método.

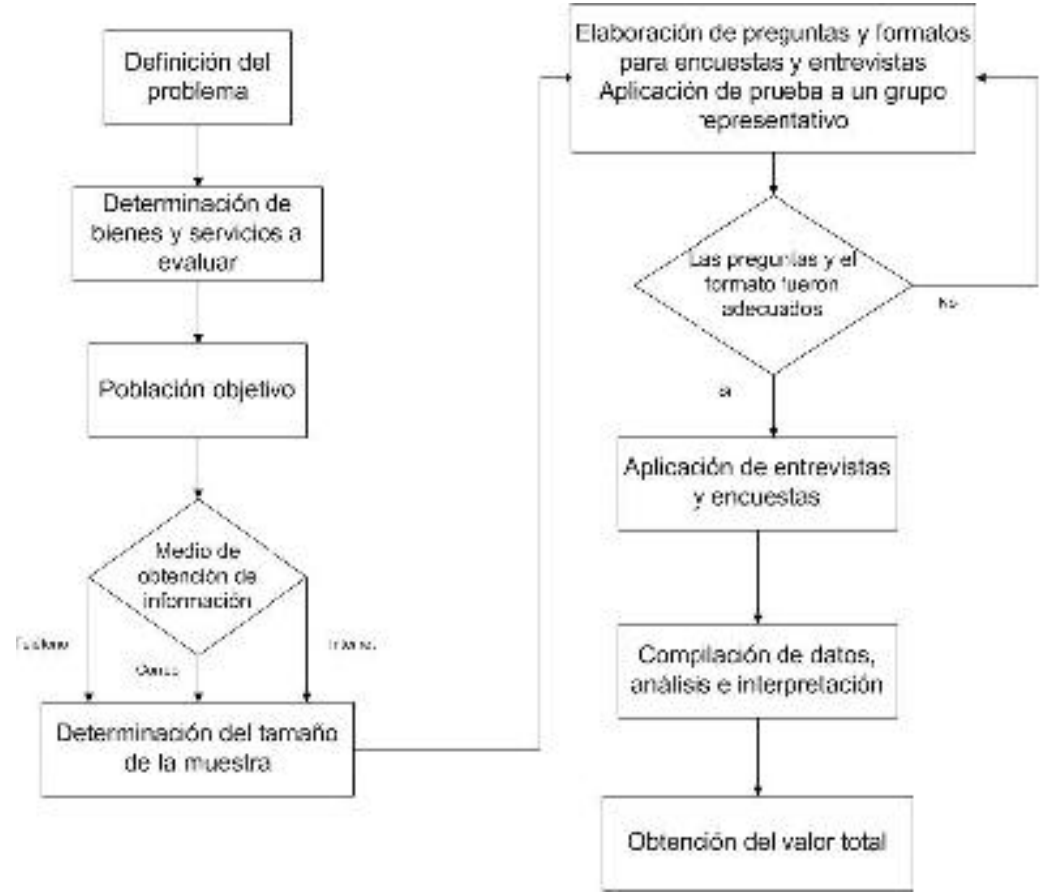

Figura 5. Diagrama de flujo del método de valoración contingente 
Métodos de valuación de externalidades ambientales provocadas por obras de ingeniería

Asimismo, es el método más utilizado en la evaluación de valores deno uso, ya que se pregunta directamente a la gente cuánto está dispuesta a pagar por servicios ambientales concretos que pueden ser afectados positiva o negativamente por una obra.

Entre los valores que pueden estimarse mediante este método pueden mencionarse los siguientes:

a) Valor económico total: la suma de todos los tipos de valores, de uso y de no uso para un bien o servicio.

b) Valor de uso: los derivados del uso actual de un bien o servicio; puede incluir, además, un uso indirecto, por ejemplo, el disfrutar un programa de televisión referente a las ballenas, el cual proporcionará un valor indirecto de uso a las mismas.

c) Valor de no uso: los que no están asociados con el uso actual o incluso la opción de utilizar un bien o servicio.

d) Valor de existencia: el que las personas depositan por el simple hecho de saber que algo existe, aun si nunca lo verán ni harán uso de él.

e) Valor de opción: el que las personas depositan por tener la opción de disfrutar de algo en el futuro, aunque esto implique no ser usado actualmente.

f) Valor de legado: el que las personas depositan en algo, sabiendo que futuras generaciones tendrán la oportunidad de disfrutarlo.

Con este método pueden conocerse las preferencias individuales en dos sentidos:

1) Cuánto se está dispuesto a pagar por algo que mejore el bienestar individual o colectivo, $y$

2) Cuánto se está dispuesto a aceptar como pago por un efecto adverso al bienestar.
Suponga que se cuenta con un parque en una comunidad. Dicho terreno es una de las alternativas para construir una plaza comercial, por lo que se requiere determinar el valor que dicho parque tiene para la población a fin de establecer si es conveniente o no construirlo en ese sitio.

Lo que va a evaluarse en este caso es el valor asignado al parque por parte de la población. Para ello, se determina una población objetivo, en donde se aplican cuestionarios y encuestas, las cuales pueden incluir preguntas como la siguiente: ¿Está usted dispuesto a pagar anualmente $\$ \mathrm{X}$ para que no se realicen obras en el sitio del parque?

Puede comenzarse con distintos valores, sea en este caso un valor de $X=\$ 10$, el cual va en aumento hasta alcanzar una cantidad que el encuestado no esté dispuesto a pagar.

Una vez que se cuenta con la información de las encuestas, se estiman los beneficios y costos. Si se emplean encuestas abiertas, preguntando cuánto están dispuestos a pagar, se puede simplemente obtener el promedio de las respuestas o usar algún método de regresión para ver cuáles factores afectan la disposición a pagar por dichos valores. Si se utilizan preguntas cerradas, cuestionando si se está dispuesto a pagar una cantidad $X$, dandole distintos valores, se obtiene el valor esperado.

De este modo, tal como lo marca el diagrama, (Figura 5) y tras el análisis de las encuestas, se llega a resultados como los mostrados en la tabla 2. Si se considera que con un costo de cero el $100 \%$ aceptaría, y se establece como máximo un valor de 250, puede obtenerse un gráfico como el de la figura 6.

La disposición promedio a pagar (lo que una persona está dispuesta a pagar en promedio por la conservación del parque), puede determinarse entonces al obtener el área bajo la curva, ya sea de manera geométrica o integrando la expresión obtenida mediante técnicas de correlación. Al multiplicarse este valor promedio por la población total involucrada, se determina el valor económico otorgado al bien evaluado. Este valor anual puede cotejarse con tra el costo de acciones de protección o sustitución para tomar la decisión final. 
Tabla 2. M étodo de valoración contingente (Disposición a pagar)

\begin{tabular}{|c|c|c|}
\hline Pregunta & $x(\$)$ & $\%$ Respuestas afirmativas \\
\hline \multirow{8}{*}{$\begin{array}{l}\text { ¿Hasta qué cantidad está dispuesto a pagar al año por conservar el } \\
\text { parque, en cuyo terreno se puede construir un centro comercial? }\end{array}$} & 10 & 95 \\
\hline & 25 & 80 \\
\hline & 50 & 60 \\
\hline & 75 & 50 \\
\hline & 100 & 25 \\
\hline & 150 & 10 \\
\hline & 200 & 5 \\
\hline & 250 & 2 \\
\hline
\end{tabular}

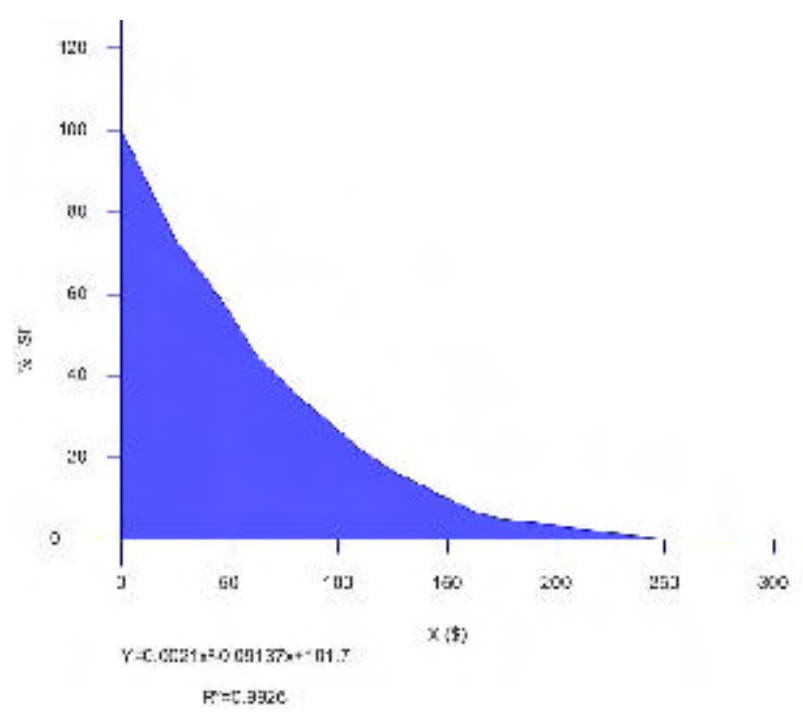

Figura 6. M étodo de valoración contingente (Disposición a pagar)

M étodo de selección contingente

Es muy similar al método de valoración contingente, permite estimar valores de prácticamente cualquier servicio o bien ambiental, además de la evaluación de valores de uso y de no uso. Se basa en la creación de escenarios hipotéticos que requieren la toma de decisiones por parte de los entrevistados.

A diferencia del método de valoración contingente, en el de selección contingente no se pide a los individuos que asignen directamente un valor monetario al bien o servicio evaluado. Estos valores se infieren mediante la elección que realiza el encuestado al presentársele distintas opciones de intercambio; por ejemplo, si prefiere un centro comercial, un conjunto habitacional, un estacionamiento, un edificio de policía o mantener el parque, etc.

\section{Métodos indirectos (de preferencias reveladas)}

M étodo del costo de viaje

Este método se basa en el comportamiento del individuo. Es muy adecuado para estimar los costos externos relacionados a sitios de recreación, aunque no exclusivamente. Asume el valor del bien ambiental al tomar en cuenta el costo que implica el acceder al sitio. Esto es, asume que el valor de dicho sitio o de los servicios que brinda se refleja en la cantidad de dinero y tiempo que está dispuesta la gente a pagar por disfrutar de ellos.

Si se analiza por individuos, define como variable dependiente el número de visitas realizadas al sitio en un período específico 
Métodos de valuación de externalidades ambientales provocadas por obras de ingeniería

$$
V=f(C, X)
$$

V Visitas al sitio

C Costo por la visita (esto incluye gastos de pasajes, uso de vehículos, combustible, peajes, tiempo de viaje, alimentos, alojamiento, etc.)

$X$ Variables socioeconómicas que influyen en el número de visitas.

Se pueden hacer también estudios por regiones, como ejemplo, véase el caso de la demanda de sitios turísticos en México. Ésta dependerá en gran parte de la accesibilidad del sitio, del medio de transporte, tiempo y costo que representa para los viajantes llegar al lugar. Por ejemplo, en Acapulco, que es uno de los sitios turísticos más importantes en donde se tienen visitantes del Distrito Federal y Estado de México en su mayoría, por la facilidad de acceso al sitio vía terrestre, considerando que la alternativa aérea generalmente no puede competir en el renglón de costos. Habitantes de regiones al Norte del país, pueden manifestar su disposición a pagar por visitar el lugar, pero ésta se incrementará considerablemente dada la distancia a recorrer, los distintos medios de transporte, así como sitios sustitutos que pueden proveer servicios similares. Estos son tan sólo algunos de los factores a considerar en este tipo de estudios, por ello, el método toma en cuenta la región de donde provienen los visitantes y la divide por zonas de origen, dejando como variable dependiente una tasa de visitas, redefiniendo la función generadora de viajes. Otro ejemplo se tiene en la figura 7 , donde el sitio se localiza en la zona Z0, y a partir de ahí se establecen las demás zonas de estudio.

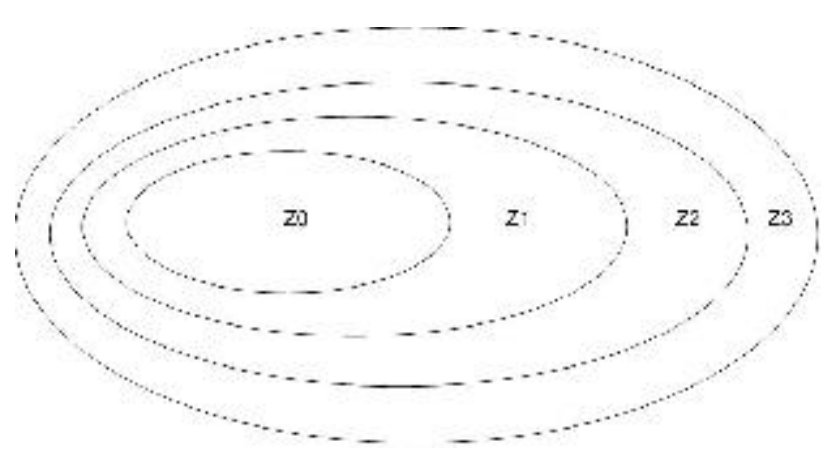

Figura 7. Zonas de estudio MCV

$$
V_{h j} / N_{h}=f\left(C_{h j}, X_{h}\right)
$$

$V_{h j}$, Visitas desde la zona $h$ al sitio $j$.

$\mathrm{N}_{h}$, Población en la zona $\mathrm{h}$.

$\mathrm{C}_{\mathrm{hj}}$, Costo por visitar el sitio j desde la zona h.

$X_{h}$, Variables socioeconómicas en la zona $h$.

Es frecuente obtener la tasa de visitantes, $V_{h j} / N_{h}$, por cada mil habitantes en la zona que corresponda.

La figura 8 muestra los pasos para la aplicación de este método. La primera etapa consiste en la obtención y análisis de la información, mientras que en la segunda se muestran los elementos a obtener del análisis.

\section{M étodo del precio hedónico}

Se basa en la suposición de que la utilidad que obtiene un individuo por un bien o servicio está en los atributos que posee dicho bien o servicio. Se separan los efectos de sus diversos atributos, de manera que pueda mostrarse cómo los cambios en los niveles de cada atributo afectan la utilidad del individuo. Para esto se obtiene una función de precio hedónico, usando técnicas de regresión múltiple con la información sobre los valores de los bienes o propiedades y sus atributos asociados. La determinación y selección de esta función influye de manera determinante en la valoración de las características analizadas. El proceso de este método se muestra en la figura 9.

\section{M étodo del precio de mercado}

Este método estima los valores económicos de servicios o productos ambientales, que son comprados y vendidos en mercados comerciales. EI método se ilustra en la figura 10.

Puede emplearse para valuar cambios la cantidad y calidad de un bien o servicio. Utiliza técnicas económicas estándar para medir los beneficios económicos de bienes del mercado. También se basa en la cantidad de personasque lo adquiere o consume, así como la cantidad suministrada a diferentes precios. 


\section{Alberto Jaime P. y R.O. Tinoco-López}
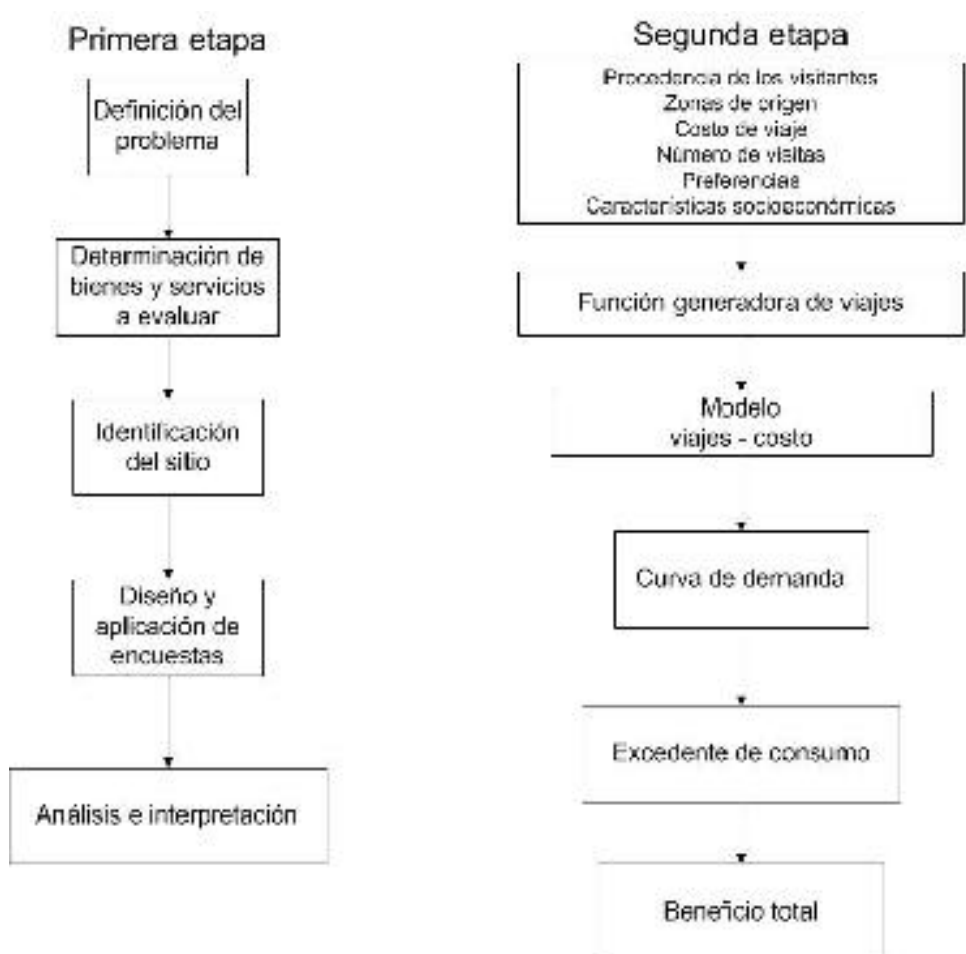

Figura 8. Diagrama del método del costo de viaje

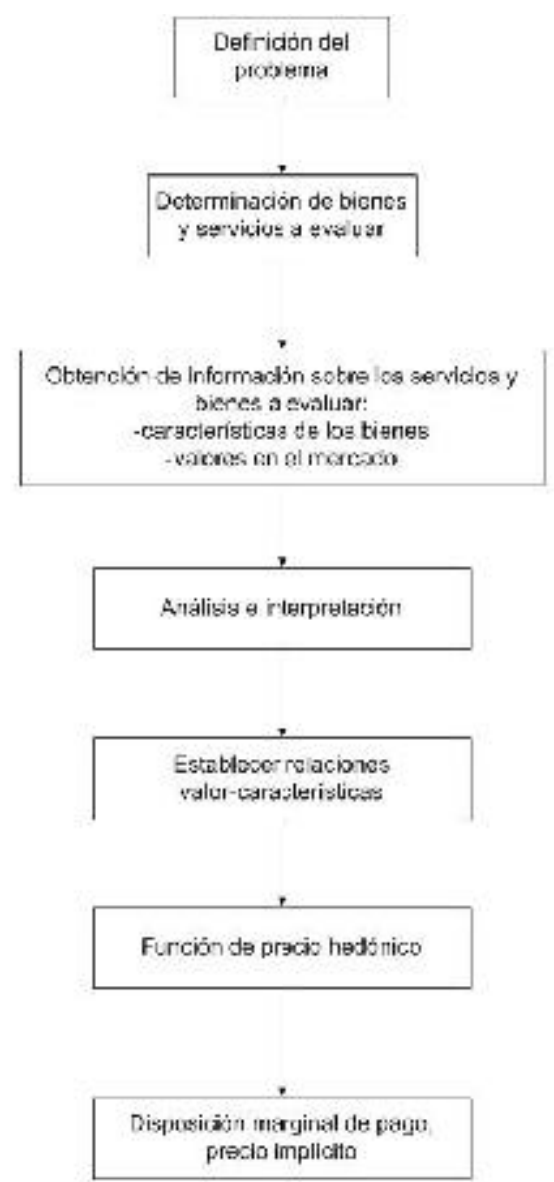

Figura 9. Diagrama de flujo del método del precio hedónico 
Métodos de valuación de externalidades ambientales provocadas por obras de ingeniería

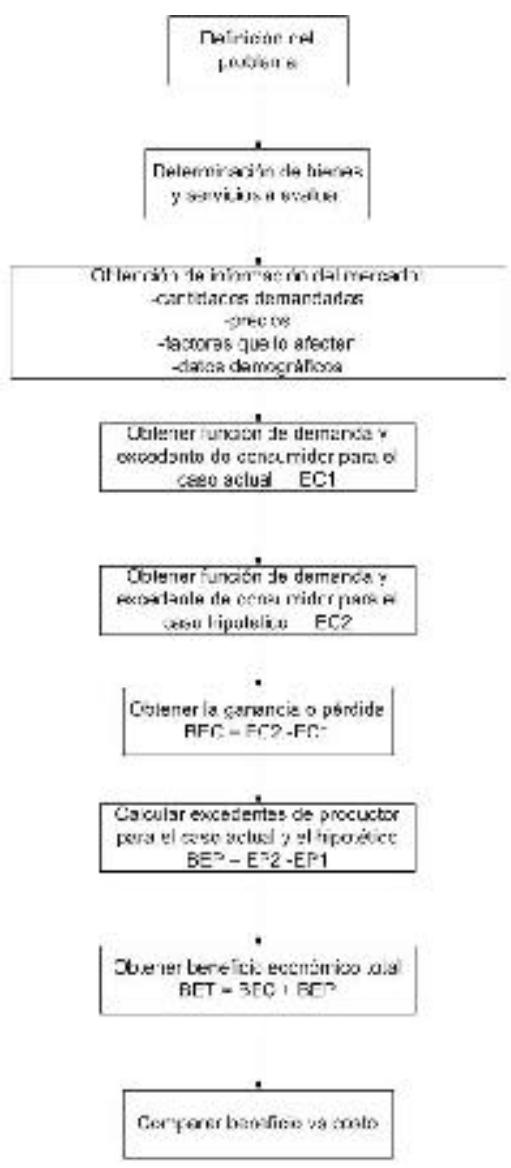

Figura 10. Diagrama de flujo del método del precio de mercado

El método estándar para medir el valor de uso de recursos que se comercian en el mercado, consiste en las estimaciones del excedente del consumidor y el excedente del productor. Para ello, emplea los precios de mercado, volúmenes de adquisición y criterios de selección, en su caso. El beneficio económico total o excedente económico, se calcula entonces al sumar los excedentes del consumidor y del productor.

\section{Otros métodos}

M étodo de dosis-respuesta

Este método estima el impacto físico que genera un cambio en el ambiente sobre un re cep tor. Esto es, establece un vínculo entre un nivel de contaminación 0 alteración al ambiente dado (dosis) y algún impacto físico (respuesta).

Puede verse como el modo más directo de valuar cambios en el ambiente, ya que consiste en la observación de los cambios físicos que sufre el mismo y estima qué alteraciones traerían dichos cambios en el valor de bienes y servicios.

Para aplicar este método deben conocerse las funciones dosis-respuesta. Se aplica principalmente en cambios al ambiente que tienen efectos en bienes comerciales. La función dosis-respuesta puede multiplicarse por un precio unitario del daño físico, a fin de dar un valor monetario del daño en cuestión mediante una función de daño económico. Los pasos a seguir son indicados en la figura 11.

M étodo de costo de reemplazo, costo de daño evitado y costo del sustituto

Los métodos de este tipo, estiman valores económicos con base en el costo, lo que implica evitar los daños resultantes de la pérdida de servicios ambientales, el costo de reemplazar dichos servicios o de proveer servicios sustitutos. 
Estrictamente, no brindan una medida de valores económicos que estarían basados en la disposición de pago por un bien o servicio. En lugar de esto, asumen que si los individuos están dispuestos a pagar por la protección o sustitución de un bien, dicho bien o servicio debe valer al menos esa cantidad. En otras palabras, con este método se estima el menor valor del bien o servicio ambiental. El método se de scribe en la figura 12.

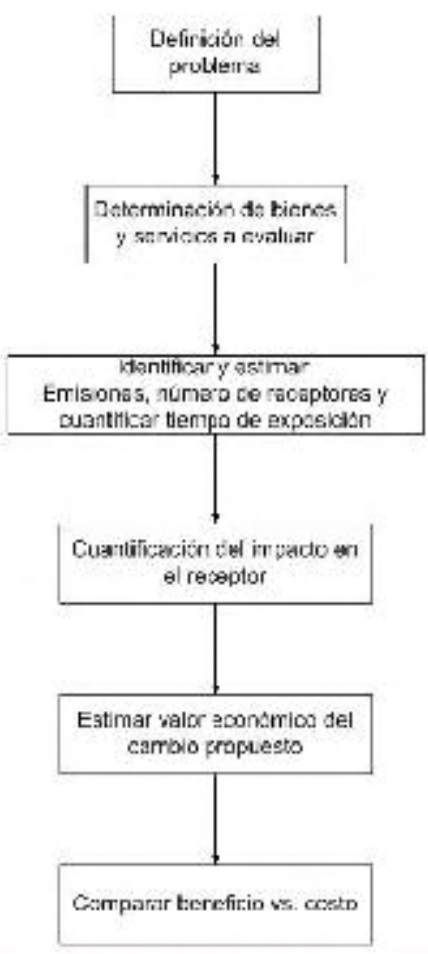

Figura 11. Diagrama de flujo del método dosis respuesta

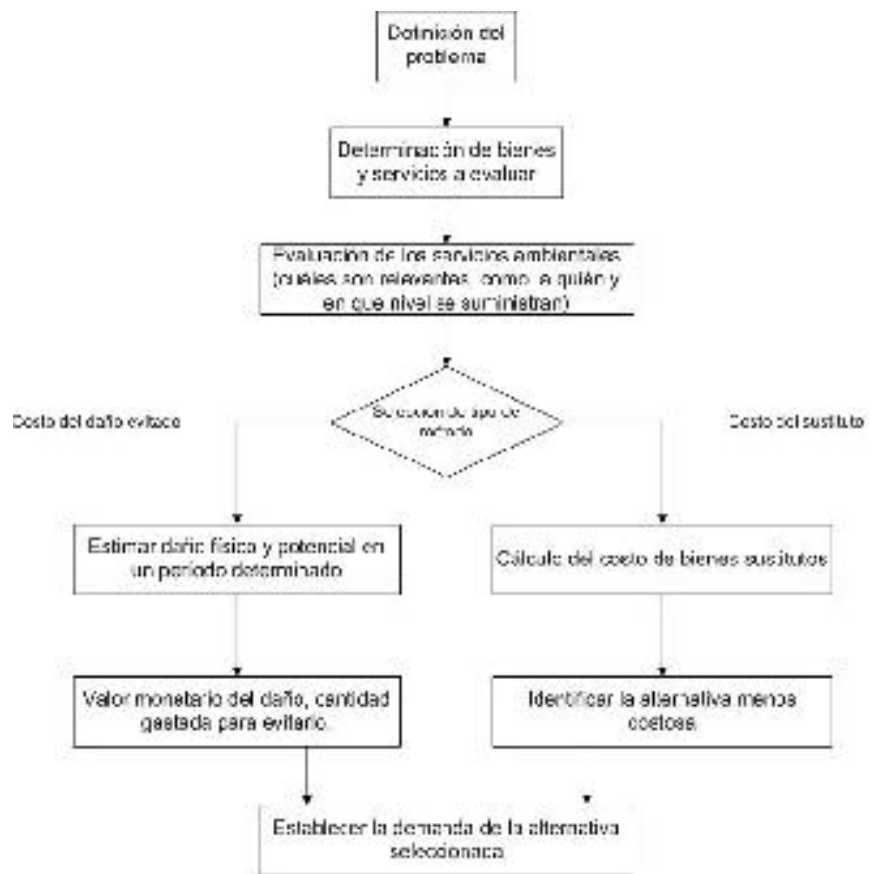

Figura 12. Diagrama de flujo del método del costo de reemplazo 
Métodos de valuación de externalidades ambientales provocadas por obras de ingeniería

\section{Internalización de externalidades}

Las externalidades (costos o beneficios) deben cargarse a aquellos responsables de las alteraciones ambientales $y$, en su caso, también a la población que disfruta de los beneficios de la obra que causa las afectaciones, de tal modo que sean consideradas en los costos del proyecto desde su planeación. Así, si las externalidades son negativas (es decir, costos), en el proyecto se consideran los gastos para evitar, disminuir o mitigar los daños causados. Asimismo, en aquellos casos donde las externalidades resultan en efectos benéficos, esta aportación también debe ser considerada, además de tomarse en cuenta en las evaluaciones correspondientes. El análisis de externalidades debe hacerse a fondo para identificar claramente en qué etapas de las obras se presentan y proponer las acciones conducentes para asignar de manera equitativa las responsabilidades por las mismas.

Para internalizar estas externalidades, pueden considerarse diversas alternativas, como los cambios en procesos, cambio de sitio o trayectoria de la obra, normas, impuestos, subsidios, así como cambios en hábitos y costumbres.

\section{Conclusiones}

La evaluación de impacto ambiental debe integrarse al proceso de planeación para decidir las mejores alternativas al realizar un proyecto 0 actividad que un promotor desee llevar a cabo (Jaime, 2003). Es decir, en la planeación de la obra 0 actividad debe incluirse la variableambiental de la misma manera que las vari ables económicas y técnicas. Posteriormente, hay que incluirla también en el análisis, diseño y construcción del proyecto. En las obras de ingeniería civil, debe tenerse clara la diferencia entre costos directos y externalidades, ya que colocarlos en su categoría correspondiente es un paso in dis pens able para su evaluación. El primer paso para evaluar externalidades ambientales es identificar y analizar los posibles impactos positivos 0 negativos que pueden provocar las obras.

Una de las dificultades para el entendimiento de externalidades, es que son costos o beneficios intangibles, es decir, lo que podría ganarse 0 perderse al realizar o no una obra de ingeniería civil, sin que esto signifique que alguien realice un desembolso o reciba una ganancia económica inmediata. Por todo esto, la percepción de las externalidades se vuelve un problema más complejo que su sola cuantificación.

No hay un método general aplicable en todos los casos. Cada problema requiere de un análisis par tic u lar en el que se deben aplicar uno o más de los métodos discutidos, se pretende tomar en cuenta la mayoría de los factores a evaluar. La adecuada combinación de estos métodos resulta entonces tan importante como la selección inicial de los mismos.

\section{Agradecimientos}

Este artículo está basado en investigaciones realizadas como parte de la tesis profesional del segundo autor bajo la dirección del primero. Se agradecen los comentarios y sugerencias de Enrique César Valdez y Oscar E. Martínez Jurado.

\section{Referencias}

Baumol W.J. and Oates W.E. ( 1988) The Theory of Environmental Policy. Cambridge, Univer sity Press.

European Commission (2003). External Costs. Euro pean Commission.

Jaime A. (2003). Las obras de ingeniería civil y su impacto ambiental. Series del Instituto de Ingeniería, UNAM, M éxico DF, SD/42.

Meade J.E. (1973). The Theory of E conomic Exter nalities. The Control of Enviromnental Pollution and Similar Social Costs. Sijthoff-Leiden, Geneva.

Ottinger R. and Wooley D. (1990). Environmental Costs of Electricity. Oceanía publi ca tions, EU.

\section{Bibliografía sugerida}

BID (2002). Funda men tals ofE nvi ron mental Impact Assessment. Trainers' Course on Environmental Management and Assessment for Investment Projects.

César E. y Vazquez A. Impacto ambiental. UNAM-IMTA.

Conesa V. (1997). Guía metodológica para la evaluación del impacto ambiental. Mundi prensa, Madrid. 
Espinoza G. (2001). Fundamentos de evaluación de impacto ambiental. BID-CED, Chile.

European Comission (1999). Externalities of Energy, Vol. 7, Euro pean Commis sion.

Field B. (1995). Economía ambiental. McGraw Hill, Colombia.

Fontaine E. (1999). Evaluación social de proyectos. Alfaomega.

Galán A. (1999). A M eth od ology Proposal to CalculatetheE xter nali ties of Liquid Biofuels. CIEMAT.

Garrod G. and Kenneth G. (2001). Economic Valu a tion of theE nvi ron ment. EE.

Lee N. and George C. (2000). Environmental Assessment in Developing and Transitional Countries. Wiley, Londres.

Muñoz F., Jiménez L. y Aguilar G. (1994). Parámetros relevantes en la evaluación de las externalidades de proyectos termoeléctricos. Instituto de Ingeniería, UNAM.

Naciones Unidas (1958). M anual de proyectos de desarrollo económico, Naciones Unidas.

Russell C. (2001). A pplying E conomics to the Environment. Oxford Univer sity Press, EUA.

Schleiner L. (1999). D ifferences in M eth od ol ogies U sed for Externality A ssess ment. RISO.

Schleiner L. (1998). Review of Externality Valua tion, RISO.

Tinoco 0. (2004). Externalidades ambientales en las obras de ingeniería civil. Tesis de licenciatura en ingeniería civil. Facultad de Ingeniería, Universidad Nacional Autónoma de México, Ciudad Universitaria, México.

\section{Semblanza de los autores}

Alberto Jaime P. Doctor en ingeniería civil por la FI deUniversidad Nacional Autónoma de México. Miembro de la International Society for Soil Mechanics and Engi neering Foun da tion y la Sociedad Mexicana de Mecánica de Suelos, asmismo, de la Academia Mexicana de Ciencias, del Colegio de Ingenieros Civiles de México, de la Sociedad de Exalumnos de la Facultad de Ingeniería y de la Academia de Ingeniería. Ha sido profesor en las divisiones profesional y de posgrado de la Facultad de Ingeniería, UNAM, y en las Universidades Iberoamericana y Autónoma del Estado de México. Ha publicado más de 80 artículos técnicos en revistas y memorias de congresos nacionales e internacionales, más de 35 informes de investigación de circulación restringida y más de 200 informes técnicos. Fue gerente de protección ambiental de la CFE (1992-1996) y subdirector general técnico de la CNA (1996-2001). Es investigador titular del Instituto de Ingeniería de la UNAM y actualmente Jefe de la División de Ingenierías Civil y Geomática de la Facultad de Ingeniería de la UNAM.

Rafael 0 mar Tinoco-López. Ingeniero Civil por la FI de la Universidad Nacional Autónoma de México, con mención honorífica, titulado bajo la dirección del Dr. Alberto Jaime P. Ha colaborado con artículos en revistas nacionales y memorias de congresos en el país. Exbecario de la División de Ingenierías Civil y Geomática de la Facultad de Ingeniería de la UNAM. 